\title{
Unconventional superconductivity and magnetism in $\mathrm{CePt}_{3} \mathrm{Si}_{1-\mathrm{x}} \mathrm{Ge}_{\mathrm{x}}$
}

\author{
E. Bauer, ${ }^{\mathrm{a}, *}$ G. Hilscher, ${ }^{\mathrm{a}}$ H. Michor, ${ }^{\mathrm{a}}$ M. Sieberer, ${ }^{\mathrm{a}}$ E.W. Scheidt, ${ }^{\mathrm{b}}$ A. Gribanov, ${ }^{\mathrm{c}}$ \\ Yu. Seropegin, ${ }^{\text {c P. Rogl, }}{ }^{\text {d A. Amato, }}{ }^{\text {e W.Y.Song, }}{ }^{\text {f,g }}$ J.-G. Park, ${ }^{\mathrm{g}}$ D.T. Adroja, ${ }^{\mathrm{f}}$ \\ M. Nicklas, ${ }^{\text {h }}$ G. Sparn, ${ }^{\text {h }}$ M. Yogi,${ }^{i}$ and Y. Kitaoka ${ }^{\mathrm{i}}$ \\ ${ }^{a}$ Institut für Festkörperphysik, Technische Universität Wien, A-1040 Wien, Austria \\ ${ }^{\mathrm{b}}$ Chemische Physik und Materialwissenschaften, Universität Augsburg, D - 86159 Augsburg, Germany \\ ${ }^{\mathrm{c}}$ Department of Chemistry, Moscow State University, Moscow, Russia \\ ${ }^{\mathrm{d}}$ Institut für Physikalische Chemie, Universität Wien, A-1090 Wien, Austria \\ e Laboratory for Muon-Spin Spectroscopy, Paul Scherrer Institute, CH-5232 Villigen PSI, Switzerland \\ f ISIS Facility, Rutherford Appleton Laboratory, Oxon OX11 OQX, England \\ $\mathrm{g}$ Department of Physics and Institute of Basic Science, Sungkyunkwan University, Suwon 440-746, Korea \\ ${ }^{\mathrm{h}}$ Max Planck Institute for Chemical Physics of Solids, D-011187 Dresden, Germany \\ ${ }^{\mathrm{i}}$ Department of Materials Science and Technology, Osaka University, Osaka 560-8531, Japan
}

\begin{abstract}
$\mathrm{CePt}_{3} \mathrm{Si}$ is a novel ternary compound exhibiting antiferromagnetic order at $T_{N} \approx 2.2 \mathrm{~K}$ and superconductivity (SC) at $T_{c} \approx 0.75 \mathrm{~K}$. Large values of $H_{c 2}^{\prime} \approx-8.5 \mathrm{~T} / \mathrm{K}$ and $H_{c 2}(0) \approx 5 \mathrm{~T}$ indicate Cooper pairs formed out of heavy quasiparticles. The mass enhancement originates from Kondo interaction with a characteristic temperature $T_{K} \approx 8 \mathrm{~K}$. NMR and $\mu \mathrm{SR}$ measurements evidence coexistence of SC and long range magnetic order on a microscopic scale. Moreover, $\mathrm{CePt}_{3} \mathrm{Si}$ is the first heavy fermion $\mathrm{SC}$ without an inversion symmetry. This gives rise to a novel type of the NMR relaxation rate $1 / T_{1}$ which is very unique and never reported before for other heavy fermion superconductors. Studies of $\mathrm{Si} / \mathrm{Ge}$ substitution allow us to establish a phase diagram.
\end{abstract}

Key words:

$\mathrm{CePt}_{3} \mathrm{Si}$, superconductivity, antiferromagnetism

Strongly correlated electron systems have been one of the most interesting topics in condensed matter physics. The key importance in such research is undoubtedly the discovery of unexpected features and new phases of metals, intermetallics and oxides at low temperatures. Among the topics, quantum phase transitions and related quantum critical phenomena are of particular importance. Quantum critical fluctuations can lead to strong renormalization of normal metallic properties as well as to novel exotic phases emerging

\footnotetext{
* Corresponding author. Tel: +43158801 13160 fax: +431 5880113199

Email address: bauer@ifp.tuwien.ac.at (E. Bauer,).
}

from these strongly fluctuating environments. One of the most exciting features in this context is the occurrence of SC.

A recently discovered example is tetragonal $\mathrm{CePt}_{3} \mathrm{Si}$ [1], the first heavy fermion SC without a centre of inversion. This implies that the electron bands are non-degenerate, except along some high-symmetry lines in the Brillouin zone [2]. Reduced degeneracy, however, weakens or even suppresses SC. Superconductivity with spin-triplet pairing should require inversion symmetry to obtain the necessary degenerate electron states [3]. Thus, it became a widespread view that a material lacking an inversion center would be an unlikely candidate for spin-triplet pairing [4]. 
Nevertheless, the extreme large value of the upper critical field $H_{c 2}(0) \approx 5 \mathrm{~T}$ of $\mathrm{CePt}_{3} \mathrm{Si}$ is inconsistent with spin-singlet Cooper-pairs, hinting at some novel features of the SC order parameter.

The aim of this paper is to provide a review of current research on $\mathrm{CePt}_{3} \mathrm{Si}$ and to locate the system in the standard generic phase diagram of heavy fermion compounds through both substitution and pressure experiments. The paper is organised as follows: After a discussion of normal state properties of $\mathrm{CePt}_{3} \mathrm{Si}$, the $\mathrm{SC}$ features of $\mathrm{CePt}_{3} \mathrm{Si}$ are examined before reporting the evolution of magnetism and $\mathrm{SC}$ in $\mathrm{CePt}_{3}(\mathrm{Si}, \mathrm{Ge})$.

Normal state properties

Physical properties of ternary $\mathrm{CePt}_{3} \mathrm{Si}$ are dominated by long range magnetic order below $T_{N} \approx 2.2 \mathrm{~K}$ and $\mathrm{SC}$ below $T_{c}=0.75 \mathrm{~K}$. Crystal electric field splitting (CEF) and Kondo interaction substantially modify Hund's $J=5 / 2$ ground state of the Ce ion. The response of the system associated with the mutual interplay of these phenomena will be highlighted below.

Fig. 1 displays $\Delta C_{p} / T$ vs. $T$ of $\mathrm{CePt}_{3} \mathrm{Si}$, where $\Delta C_{p}$ is defined by the difference between the $C_{p}(T)$ data of $\mathrm{CePt}_{3} \mathrm{Si}$ and $\mathrm{LaPt}_{3} \mathrm{Si}$, i.e. $C_{m a g} \sim \Delta C_{p}$. This plot exhibits three distinct features: i) the $\mathrm{SC}$ transition of $\mathrm{CePt}_{3} \mathrm{Si}$ at $T_{c}=0.75 \mathrm{~K}$ (see below), ii) a magnetic transition at $T_{N} \approx 2.2 \mathrm{~K}$ and iii) an almost logarithmic tail of $\Delta C_{p} / T$ above $T_{N}$, stretching roughly up to $10 \mathrm{~K}$. Well above $10 \mathrm{~K}$, Schottky contributions dominate in the specific heat. The integrated entropy up to $20 \mathrm{~K}$ is nearly $R \ln 2$, and the entropy of $8.7 \mathrm{~J} / \mathrm{mol}-\mathrm{K}$ integrated up to $100 \mathrm{~K}$ is slightly less than $R \ln 4=$ $11.5 \mathrm{~J} / \mathrm{mol}-\mathrm{K}$. These results clearly indicate that the ground state of $\mathrm{Ce}^{3+}$ ions is a doublet with the first excited level above $100 \mathrm{~K}$. The logarithmic temperature dependence observed just above the magnetic transition may be considered as hint of non-Fermi liquid behaviour. Therefore, it is a unique observation that nonFermi liquid behaviour, magnetic ordering and eventually a SC transition consecutively arises on the same sample upon lowering temperature.

In order to analyze in more detail the magnetically ordered region of the system, a model by Continentino [5] is applied with the following analytic expression for the specific heat well below $T_{m a g}$ :

$$
\begin{aligned}
C_{\text {mag }} & =g \Delta^{7 / 2} T^{1 / 2} \exp (-\Delta / T) \\
& \times\left[1+\frac{39}{20}\left(\frac{T}{\Delta}\right)+\frac{51}{32}\left(\frac{T}{\Delta}\right)^{2}\right],
\end{aligned}
$$

This expression is based on antiferromagnetic magnons with a dispersion relation given by $\omega=\sqrt{\Delta^{2}+D^{2} k^{2}}$, where $\Delta$ is the spin-wave gap and $D$ is the spin-wave

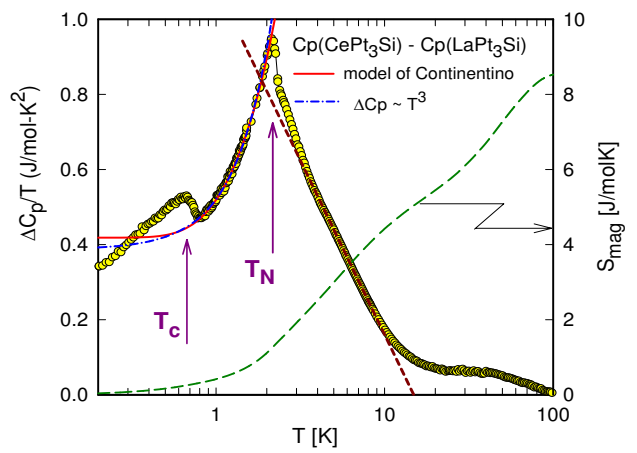

Fig. 1. Temperature dependent magnetic contribution to the specific heat, $\Delta C_{p}$ of $\mathrm{CePt}_{3} \mathrm{Si}$ plotted as $\Delta C_{p} / T$ on a logarithmic temperature scale. The long-dashed line represents the magnetic entropy (right axis). The short-dashed line is a guide to the eyes and roughly indicates the non-Fermi liquid behaviour. The solid line is a fit according to Eq. 1 and the dashed-dotted line is a fit according to $\Delta C_{p} \sim T^{3}$.

velocity; $g \propto 1 / D^{3} \propto 1 / \Gamma^{3}$ and $\Gamma$ is an effective magnetic coupling between Ce ions. A least squares fit of Eq. 1 to the data below $T_{N}$ (solid line, Fig. 1) reveals $\Delta \approx 2.7 \mathrm{~K}$, a reasonable gap value with respect to the ordering temperature. Another model calculation with simple antiferromagnetic spin waves with $C_{m a g} \propto T^{3}$ gives reasonable agreement with the data, too. A recent neutron diffraction study on $\mathrm{CePt}_{3} \mathrm{Si}$ reveals antiferromagnetic ordering below $T_{N} \approx 2.2 \mathrm{~K}$ with a wave vector $\mathbf{k}=(0,0,1 / 2)$, i.e. doubling of the magnetic unit cell along c-direction [6]. Using both models, we estimate a Sommerfeld coefficient of 0.41 and $0.39 \mathrm{~J} / \mathrm{molK}^{2}$ for the former and latter models, respectively. These figures are in good agreement with an extrapolation of high field specific heat data where SC is suppressed by applying magnetic fields.

Considering Kondo type interactions to be responsible for the significant renormalisation of electrons in $\mathrm{CePt}_{3} \mathrm{Si}$ as evidenced by the Sommerfeld value $\gamma$, the magnetic entropy allows to estimate the Kondo temperature $T_{K}$. Taking the results derived in Ref. [7] yields $T_{K} \approx 7.2 \mathrm{~K}$. A second possible estimate $T_{K}$ follows from the competition of the RKKY interaction and the Kondo effect, which leads to a significant reduction of the specific heat jump at $T=T_{N}$. Following the procedure developed in Ref. [8] gives $T_{K} \approx 9 \mathrm{~K}$, in reasonable agreement with the previous estimate.

For better understanding of the magnetic ground state and expected localized character of Ce $4 \mathrm{f}$ electrons, inelastic neutron scattering experiments at the HET spectrometer of ISIS, UK, were carried out. In order to accurately and reliably determine magnetic scattering from the magnetic moments of Ce, both $\mathrm{CePt}_{3} \mathrm{Si}$ and $\mathrm{LaPt}_{3} \mathrm{Si}$ were investigated in powder form under identical conditions. For phonon sub- 
traction, we used two well-established methods [9], with almost the same results. Our main finding is that there are strong and clear features from 10 to $30 \mathrm{meV}$. As shown in Fig. 2 ought to be of magnetic

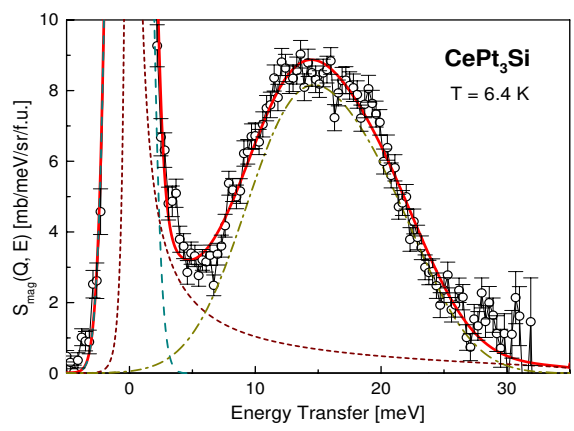

Fig. 2. Magnetic scattering obtained at $6.4 \mathrm{~K}$ with the incident energy of $35 \mathrm{meV}$. The dashed line is for the elastic component with $\mathrm{FWHM}=2.4 \mathrm{meV}$ while the short-dashed line represents the quasi-elastic component with FWHM=0.8 meV. The dashed-dotted line is for the sum of two Lorentzian components centered at 13 and $20 \mathrm{meV}$ with $\mathrm{FWHM}=10.0 \mathrm{meV}$.

origin as the phonon contributions have been subtracted from the data. In order to explain the data, we used the CEF Hamiltonian for $\mathrm{Ce}^{3+}$ with $\mathrm{C}_{4 v}$ point symmetry: $H_{C E F}=B_{2}^{0} O_{2}^{0}+B_{4}^{0} O_{4}^{0}+B_{4}^{4} O_{4}^{4}$. With $B_{2}^{0}=-0.4972 \mathrm{meV}, B_{4}^{0}=0.0418 \mathrm{meV}$, and $B_{4}^{4}=0.2314$ $\mathrm{meV}$, the observed magnetic scattering is reasonably well explained (solid line, Fig. 2). Keeping the CEF parameters unchanged, we could also explain the data obtained at $94 \mathrm{~K}$ equally well. Moreover, the two CEF excitations centred at 13 and $20 \mathrm{meV}$ are also consistent with the heat capacity data as discussed above. We furthermore studied low energy excitations using lower incident energy to find a weak feature around $1.4 \mathrm{meV}$. The dispersion of that intensity at $T=5 \mathrm{~K}$, particularly around $Q=0.8 \AA^{-1}$ is a signature for the development of short-ranged magnetic correlations and explains the anomalous behaviour of the specific heat above magnetic ordering. At higher temperatures $(T \approx 30 \mathrm{~K})$ scattering becomes $Q$-independent. This feature is completely absent in non-magnetic $\mathrm{LaPt}_{3} \mathrm{Si}$. We note that a recent inelastic neutron scattering experiment [6] reported two CEF peaks at 1.0 and $24 \mathrm{meV}$. However, our data show that their $2 \mathrm{nd}$ excitation is most likely to be mistaken.

Superconducting properties of $\mathrm{CePt}_{3} \mathrm{Si}$

Signs of bulk SC of $\mathrm{CePt}_{3} \mathrm{Si}$ below $T_{c}=0.75 \mathrm{~K}$ are numerous: zero resistivity, diamagnetic signal in the susceptibility, a jump in the specific heat and NMR relaxation rate at $T_{c}$. Here, we show in Fig. 3 (a) the field dependent specific heat at low temperatures and at various external magnetic fields. Sommerfeld coeffi-

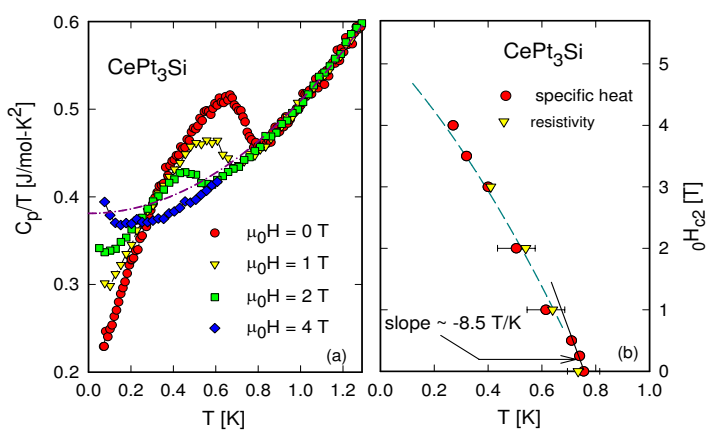

Fig. 3. (a): Temperature dependent specific heat $C_{p} / T$ of $\mathrm{CePt}_{3} \mathrm{Si}$ for various values of applied fields; the dashed line is a $T^{3}$ extrapolation of $C_{p}(T)$ at $0 \mathrm{~T}$. (b): Temperature dependence of the upper critical field $H_{c 2}$. The solid straight line yields $H_{c 2}^{\prime} \approx-8.5 \mathrm{~T} / \mathrm{K}$; the dashed line is a guide to the eyes.

cient $\gamma_{n} \approx 0.39 \mathrm{~J} / \mathrm{molK}^{2}$ of $\mathrm{CePt}_{3} \mathrm{Si}$ at zero field can be obtained from a careful extrapolation of the normal state invoking the $T^{3}$-dependence associated with antiferromagnetic ordering. This extrapolation also satisfies the basic requirement of an entropy balance between the $\mathrm{SC}$ and normal state regions.

The application of magnetic fields reduces $T_{c}$, giving rise to a rather large change of $d H_{c 2} / d T \equiv H_{c 2}^{\prime} \approx$ $-8.5 \mathrm{~T} / \mathrm{K}$, in good agreement with the conclusion drawn from electrical resistivity [see Fig. 3(b)]. An extrapolation of $T_{c}(H)$ towards zero yields $H_{c 2}(0) \approx 5 \mathrm{~T}$, well above the Pauli - Clogston limiting field [1]. Furthermore, an estimation of the Sommerfeld coefficient from the high field data gives $0.36 \mathrm{~J} / \mathrm{molK}^{2}$, in fair agreement with the value obtained from an extrapolation of the normal state in the zero field data (see Fig. 3 ). The upturn of $C_{p} / T$ at lowest temperatures that gets stronger with increasing magnetic fields is most likely due to the nuclear contribution of ${ }^{195} \mathrm{Pt}$.

The jump in the specific heat $\Delta C_{p} /\left.T\right|_{T_{c}} \approx$ $0.1 \mathrm{~J} / \mathrm{molK}^{2}$, leads to $\Delta C_{p} /\left(\gamma_{n} T_{c}\right) \approx 0.25$, which is much smaller than expected from the BCS theory $\left(\Delta C_{p} /\left(\gamma T_{c}\right) \approx 1.43\right)$. Even using the electronic specific heat coefficient in the SC state, $\gamma_{s} \approx 0.18(1) \mathrm{J} / \mathrm{molK}^{2}$, we obtained $\Delta C_{p} /\left(\gamma_{s} T_{c}\right) \approx 0.55$ that is still below the $\mathrm{BCS}$ value.

We can think of two scenarios that may well explain such a substantial reduction of $\Delta C_{p} / \gamma T_{c}$ with respect to the BCS value; i) spin triplet SC like $\mathrm{Sr}_{2} \mathrm{RuO}_{4}$ exhibit a similarily reduced magnitude of $\Delta C_{p} /\left(\gamma T_{c}\right)[10]$ and ii) not all electrons condense into Cooper pairs; thus only a fraction of the carriers mediate the supercurrent. It implies that electrons responsible for nor- 
mal state features, like antiferromagnetic order, coexist with those forming the Cooper pairs. In fact, the finite value of $\gamma_{s} \approx 0.18 \mathrm{~J} / \mathrm{molK}^{2}$ provides evidence that even at $T=0 \mathrm{~K}$ a significant portion of the Fermi surface is still not involved in the SC condensate.

Microscopic evidence for the latter conclusion can be found from zero-field $\mu \mathrm{SR}$ spectroscopy data obtained in the magnetic phase below and above $T_{c}$ in the magnetic phase. (Fig. 4). At temperatures much above $T_{N}$, the $\mu \mathrm{SR}$ signal is characteristic of a paramagnetic state with a depolarization solely arising from nuclear moments. Below $T_{N}$ the $\mu \mathrm{SR}$ signal indicates that the full sample volume orders magnetically. High statistic runs performed above and below $T_{c}$ did not show any change of the magnetic signal, supporting the view of a microscopic coexistence between magnetism and SC. This points to a novel state for SC Ce-based

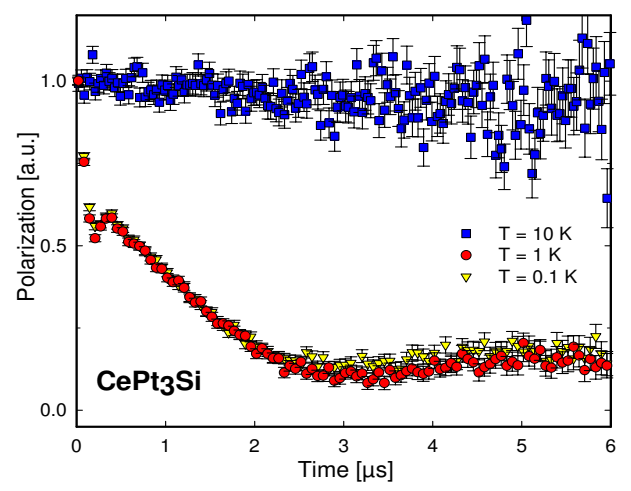

Fig. 4. Zero field depolarisation rate of $\mathrm{CePt}_{3} \mathrm{Si}$ at various temperatures.

heavy-fermion systems at ambient pressure, for which, to date, magnetism was found to be either absent [11] or strongly competing against SC [12]. The observed coexistence is reminiscent of the situation observed in $\mathrm{UPd}_{2} \mathrm{Al}_{3}$ [13], where a model of two independent electron subsets, localized or itinerant, was proposed in view of similar microscopic data [14].

Another microscopic information about the SC state can be obtained from the temperature dependent ${ }^{195} \mathrm{Pt}$ nuclear spin-relaxation rate $1 / T_{1}[15]$. Results are shown as $\left(1 / T_{1} T\right)_{\mathrm{SC}} /\left(1 / T_{1} T\right)_{\mathrm{N}}$ vs. $T / T_{\mathrm{c}}$ plot in Fig. 5 for 8.9 and $18.1 \mathrm{MHz}$. The relaxation behaviour $1 / T_{1} T$ of $\mathrm{CePt}_{3} \mathrm{Si}$ is characterized by a kind of the Hebel-Slichter anomaly [16] indicating coherence effects as in conventional BCS SC. The peak height, however, is significantly smaller than that observed for conventional BCS SC and, additionally, shows no field dependence at the $8.9 \mathrm{MHz}(H \sim 1 \mathrm{~T})$ and 18.1 $\mathrm{MHz}(H \sim 2 \mathrm{~T})$ run.

$\left(1 / T_{1} T\right)$ at $H \sim 2 \mathrm{~T}$ seems to saturate at low temperature, which can be attributed to the presence of vortex cores where the normal-state region is intro- duced. $1 / T_{1} T$ at $8.9 \mathrm{MHz}(H \sim 1 \mathrm{~T})$, however, continues to decrease down to $T=0.2 \mathrm{~K}$, the lowest measured temperature. Neither an exponential law nor a $T^{3}$ behaviour is observed for the data down to $T=0.2$ $\mathrm{K}$. Therefore, $\mathrm{CePt}_{3} \mathrm{Si}$ is the first $\mathrm{HF} \mathrm{SC}$ that exhibits a peak in $1 / T_{1} T$ just below $T_{\mathrm{c}}$ and, moreover, does not follow the $T^{3}$ law reported for most of the unconventional HF SC (see e.g., Ref. [17] and Refs. therein).

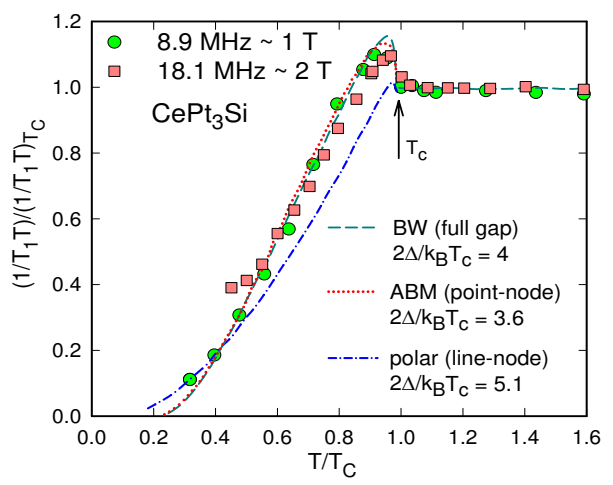

Fig. 5. A plot of $\left(1 / T_{1} T\right) /\left(1 / T_{1} T\right)_{T_{c}}$ vs. $T / T_{\mathrm{c}}$ at $8.9 \mathrm{MHz}$ $(H \sim 1 \mathrm{~T})$ and $18.1 \mathrm{MHz}(H \sim 2 \mathrm{~T})$. The dashed line is for the Balian-Werthamer model (BW isotropic triplet SC state) with a value of $2 \Delta / k_{\mathrm{B}} T_{\mathrm{c}}=4$. The dotted line assumes a point-node model with $2 \Delta / k_{\mathrm{B}} T_{\mathrm{c}}=3.6$ and the dashed-dotted line represents a fit by a line-node gap model with $2 \Delta / k_{\mathrm{B}} T_{\mathrm{c}}=5.1$.

To account for the relaxation behavior below $T_{\mathrm{c}}$ in noncentrosymmetric $\mathrm{CePt}_{3} \mathrm{Si}$, three models were adopted for a description of the temperature dependence of $1 / T_{1}$ at $H \sim 1 \mathrm{~T}$. The dashed line in Fig. 5 represents a fit according to the the Balian-Werthamer model (isotropic spin-triplet $\mathrm{SC}$ state) with a value of $2 \Delta / k_{\mathrm{B}} T_{\mathrm{c}}=3.9$ [18], while the dashed-dotted line is a fit using a line-node model with $2 \Delta / k_{\mathrm{B}} T_{\mathrm{c}}=5.1$. The dotted line refers to a point-node model with $2 \Delta / k_{\mathrm{B}} T_{\mathrm{c}}=3.6$. The models used, however, failed to give satisfactory description of the observed temperature dependence of $1 / T_{1}$ over the entire temperature range. While the line-node model gives a reasonable agreement with the data at lowest temperatures, the BW model describes reasonably well the data just below $T_{\mathrm{c}}$. The peak in $1 / T_{1} T$ would indicate the presence of an isotropic energy gap, even though a coherence effect - inherent for the isotropic spin-singlet s-wave pairing state - is absent.

In almost all previous studies on either conventional and unconventional SC, it was assumed that the crystal has an inversion center, which allows separate consideration of the even (spin-singlet) and odd (spin-triplet) components of the $\mathrm{SC}$ order parameter. In $\mathrm{CePt}_{3} \mathrm{Si}$, however, a center of symmetry is absent. Therefore, the novel relaxation behaviour found below $T_{\mathrm{c}}$ hints at a possibly new class of a $\mathrm{SC}$ state being realized in noncentrosymmetric $\mathrm{CePt}_{3} \mathrm{Si}$. 
Gor'kov and Rashba [19] demonstrated that in the absence of inversion symmetry the order parameter becomes a mixture of spin-singlet and spin-triplet components, which leads, for instance, to the Knight shift attaining a non- zero value at $T=0 \mathrm{~K}$. A novel idea with respect to the order parameter of systems without inversion symmetry was put forward very recently by Saxena and Monthoux [20]. In their model for the case of broken inversion symmetry, the spins might rotate in the momentum space around the surface.

Evolution of magnetism and superconductivity in $\mathrm{CePt}_{3}(\mathrm{Si}, \mathrm{Ge})$

In order to follow the evolution of physical properties upon $\mathrm{Si} / \mathrm{Ge}$ substitution, we prepared several alloys. Guinier X-ray powder intensity profiles of the alloys from the series $\mathrm{CePt}_{3}\left(\mathrm{Si}_{1-\mathrm{x}} \mathrm{Ge}_{\mathrm{x}}\right)$ with $x=$ $0.02,0.03,0.06,0.10,0.15$ and 0.20 were all indexed completely on the basis of a primitive tetragonal unit cell, confirming isotypism with the structure type of $\mathrm{CePt}_{3} \mathrm{Si}[21]$ with a random substitution of the $\mathrm{Si} / \mathrm{Ge}$ atoms in the $1 a$ sites of space group $P 4 / \mathrm{mmm}$. Small secondary peaks arise beyond $x \approx 0.2$ indicating the limit of the $\mathrm{CePt}_{3} \mathrm{~B}$ type phase region. Figure 6 shows

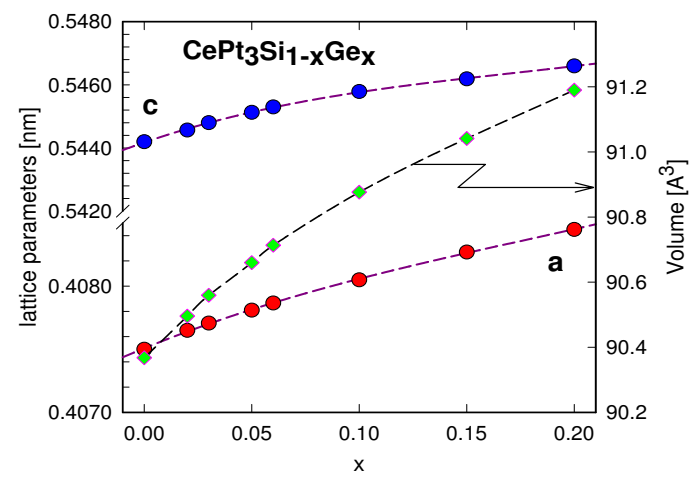

Fig. 6. Concentration dependent lattice parameters $a, c$ and unit cell volume $V$ of $\mathrm{CePt}_{3} \mathrm{Si}_{1-\mathrm{x}} \mathrm{Ge}_{\mathrm{x}}$.

the concentration dependent lattice parameters $a$ and $c$ as a function of $\mathrm{Si} / \mathrm{Ge}$ substitution, together with the unit cell volume. The monotonic increase of both $a$ and $c$ parameters yields a growing unit cell volume upon doping. The general increase of the unit cell volumes reduces chemical pressure on the Ce atoms leading to a decrease of hybridization.

The temperature dependent electrical resistivity $\rho$ of $\mathrm{CePt}_{3}\left(\mathrm{Si}_{1-\mathrm{x}} \mathrm{Ge}_{\mathrm{x}}\right)$ is shown for several concentrations $x$ in Fig. 7 for temperatures below about $4 \mathrm{~K}$. The overall $\rho(T)$ values increase due to increasing substitutional disorder on the $1 a$ site. Samples investigated

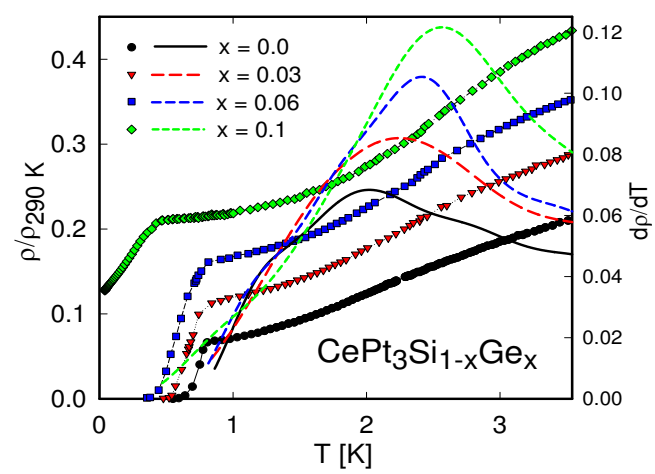

Fig. 7. Low temperature electrical resistivity $\rho$ for various concentrations $x$ of $\mathrm{CePt}_{3} \mathrm{Si}_{1-\mathrm{x}} \mathrm{Ge}_{\mathrm{x}}$ (symbols), and temperature derivative $\mathrm{d} \rho / \mathrm{d} T$ (lines, right axis).

are characterized by a concentration dependent onset of $\mathrm{SC}$, where the $\mathrm{Si} / \mathrm{Ge}$ substitution suppresses the $\mathrm{SC}$ roughly above $x=0.1$. At somewhat elevated temperatures, $\rho(T)$ exhibits a pronounced curvature which can be taken as a signature for long range magnetic order, in agreement with specific heat measurements. A magnetic instability is evidenced from the resistivity data in terms of a $\mathrm{d} \rho / \mathrm{d} T$ plot (lines, Fig. 7 , right axis). $\mathrm{d} \rho / \mathrm{d} T$ shows pronounced anomalies, referring to broadened magnetic phase transitions; the respective temperatures increase continuously with increasing Ge content.

The magnetic contribution to the specific heat of $\mathrm{CePt}_{3}\left(\mathrm{Si}_{1-\mathrm{x}} \mathrm{Ge}_{\mathrm{x}}\right)$ is plotted in Fig. 8 as $C_{m a g} / T$ vs. $T$ for several compositions up to $x=0.2$ from 1.8 to 20 K. $C_{m a g}$ is derived as in Fig. 1 using the measured data of isostructural non-magnetic $\mathrm{LaPt}_{3} \mathrm{Si}$. A com-

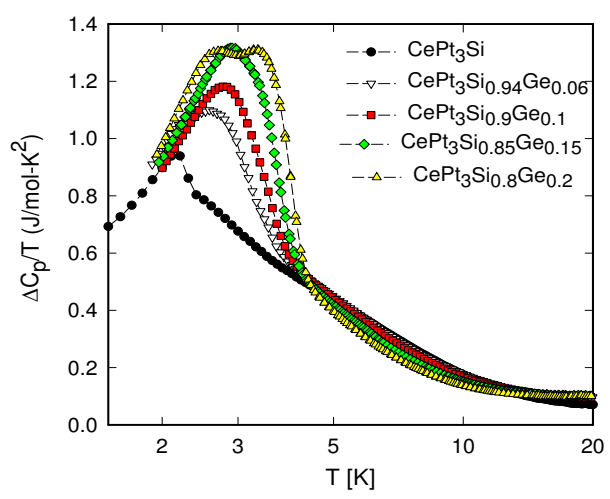

Fig. 8. Magnetic contribution to the specifc heat $\Delta C_{p}$ plotted as $C_{p} / T$ vs. $T$ for various alloys of $\mathrm{CePt}_{3} \mathrm{Si}_{1-\mathrm{x}} \mathrm{Ge}_{\mathrm{x}}$.

mon feature in all the data-sets is a mean-field like anomaly, which is associated with magnetic order of the cerium sublattice. $T_{N}$ rises with increasing Ge content and, simultaneously, the phase transitions appear to be broadened, in agreement with the observation 
made from $\mathrm{d} \rho / \mathrm{d} T$. While for $x=0$, the logarithmic behaviour well above $T_{N}$ is quite pronounced and obvious, its temperature range becomes narrower with increasing Ge content as a consequence of increasing magnetic interactions, driving a magnetic instability at much higher temperatures. The double-peak feature for $x=0.2$ is most likely due to a secondary phase as indicated by our XRD analysis.

The phase diagram shown in Fig. 9 summarizes characteristic temperatures deduced for $\mathrm{CePt}_{3}\left(\mathrm{Si}_{1-\mathrm{x}} \mathrm{Ge}_{\mathrm{x}}\right)$ at ambient pressure, as well as data derived from resistivity studies on $\mathrm{CePt}_{3} \mathrm{Si}$ under hydrostatic pressure up to about $15 \mathrm{kbar}$ [22]. In order to make comparison between substitution and pressure, Murnaghan's equation of state is adopted, with a bulk modulus $B_{0}=$ 1000 kbar. Pressure of 15 kbarcorresponds then with a reduction of the unit cell volume of about $1 \%$. The

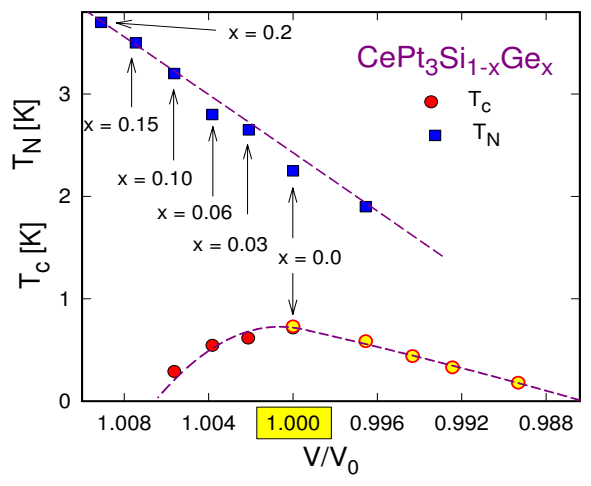

Fig. 9. Phase diagram of $\mathrm{CePt}_{3}\left(\mathrm{Si}_{1-\mathrm{x}} \mathrm{Ge}_{\mathrm{x}}\right)$.

phase diagram thus deduced resembles very well those characteristics, which determine the standard generic phase diagram associated with a quantum phase transition. The increase of the unit cell volume by the $\mathrm{Si} / \mathrm{Ge}$ substitution is, in a first-order approximation, responsible for a decrease of hybridisation. This has two consequences: i) the Kondo interaction decreases and ii) magnetic interaction strengthens, causing the observed increase of the magnetic transition temperature. Concomitantly, the SC transition temperature becomes suppressed and finally, $\mathrm{SC}$ vanishes beyond $x>0.1$. Increasing hydrostatic pressure is responsible for a decrease of both $T_{N}$ and $T_{c}$. Ternary $\mathrm{CePt}_{3} \mathrm{Si}$ thus appears to be, by chance, situated at the maximum position of the "superconducting dome". Whether or not this SC dome is constrained within the magnetic phase is still unknown. Depending on the particular choice of $B_{0}$, i.e. smaller or larger than $B_{0}=1000 \mathrm{kbar}$ the volume below $V / V_{0}=1$ becomes strechted or narrower. In any case, $T_{c}^{\max }$ is well below the magnetic phase line, being a signature that Cooper pairing may be mediated by magnetic fluctuations rather than by the standard phonon mechanism.
We summarize that non-centrosymmetric $\mathrm{CePt}_{3} \mathrm{Si}$ is a heavy fermion $\mathrm{SC}$ with $T_{c}=0.75 \mathrm{~K}$ that orders magnetically at $T_{N}=2.2 \mathrm{~K}$. The NMR relaxation rate $1 / T_{1}$ shows unexpected features which were found before neither in conventional nor in heavy fermion SC, indicative of very unusual shapes of the SC order parameter. In fact, a number of theoretical scenarios support these observations. The $\mathrm{Si} / \mathrm{Ge}$ substitution simply drives a volume expansion, thus magnetism is stabilized and SC finally ceases to exist.

Work supported by the Austrian FWF P16370, P15066 and by the DFG, SFB 484 (Augsburg). The Austrian - Russian exchange within project I.18/4 is acknowledged. JGP and WYS acknowledge financial support by the CSCMR of Seoul National University.

References

[1] E. Bauer et al., Phys. Rev. Lett. 92 (2004) 027003.

[2] K.V. Samokhin et al., Phys. Rev. B 69 (2004) 094514.

[3] P.W. Anderson, Phys. Rev. B 30 (1984) 4000.

[4] P. Frigeri et al., Phys. Rev. Lett. 92 (2004) 097001.

[5] M. Continentino et al., Phys. Rev. B 64 (2001) 012404.

[6] N. Metoki et al., J. Phys.: Cond. Mat. 16 (2004) L207.

[7] H.U. Desgranges and K.D. Schotte, Phys. Lett. A 91 (1982) 240.

[8] M. Besnus et al., J. Magn. Magn. Mat. 104 - 107 (1992) 1385.

[9] D. T. Adroja et al., Phys. Rev. B68 (2003) 094425.

[10] A.P. Makenzie and Y. Maeno, Rev. Mod. Phys. 75,(2003) 657 .

[11] C. Petrovic et al., Europhys. Lett. 53 (2001) 354.

[12] G.M. Luke et al., Phys. Rev. Lett. 73 (1994) 1853.

[13] R. Feyerherm et al., Phys. Rev. B 56 (1997) 699.

[14] R. Caspary et al., Phys. Rev. Lett. 71 (1993) 2146.

[15] M. Yogi et al., Phys. Rev. Lett. (2004) in press.

[16] L.C.Hebel and C.P. Slichter, Phys. Rev. 107 (1957) 901.

[17] H. Tou et al., J. Phys. Soc. Jpn. 64 (2003) 725.

[18] R. Balian and N. R. Werthamer, Phys. Rev. 131 (1963) 1553.

[19] L.P. Gorkov and E.I. Rashba, Phys. Rev. Lett. 87 (2001) 037004.

[20] S.S. Saxena and P. Monthoux, Nature 427 (2004) 799.

[21] O. Sologub et al., J. Alloys Compounds 337 (2002) 10.

[22] M. Nicklas, et al., to be presented at this conference. 